percentage accuracy was observed between the control and nap-opportunity groups $(\mathrm{p}=0.31)$.

Conclusions The main finding of this study was that the sleep-deprived group derive greater benefit in cognitive function from a 20-minute nap-opportunity. Future studies could investigate the relationship between the amount of sleep-deprivation and the magnitude of benefit derived from a nap-opportunity. Moreover, the impact of napping in individuals who are chronically sleep-deprived should also be explored.

\section{P023 A SYSTEMATIC REVIEW AND META-ANALYSIS OF PHARMACOLOGICAL INTERVENTIONS FOR SLEEP PROBLEMS IN CHILDREN AND ADOLESCENTS WITH AUTISM SPECTRUM DISORDER}

${ }^{1}$ Raghad Alonazi, ${ }^{1}$ Rahaf Alonazi, ${ }^{1}$ Amna Al-Ahmed, ${ }^{1}$ Hajar Arnous, ${ }^{2}$ Faisal Alotaibi, ${ }^{3}$ Hetaf Alammar*. ${ }^{1}$ Medical intern at Dar Al Uloom University, Riyadh; ${ }^{2}$ Paediatrician at King Fahad Medical City, Riyadh; ${ }^{3}$ Assistant professor in psychology at Shaqra University, Shaqra

\subsection{6/bmiresp-2019-bssconf.23}

Aim The aim of our systematic review and meta-analysis was to synthesise the high-quality evidence on the efficacy and safety of pharmacological interventions to manage sleep problems in children and adolescents with autism spectrum disorder (ASD).

Methods The studies we included here were (RCTs) looking at the efficacy and safety of pharmacological intervention for sleep problems in children and adolescents with ASD. The studies had to include objective sleep measures and/or subjective sleep measures. They all dealt with pharmacological interventions, with medications including melatonin and antipsychotics, and they all compared these drugs with placebos. We used four databases: ERIC, ProQuest, PubMed and Ovid Medline. We assessed the papers in accordance with the Cochrane risk of bias tool.

Results We found six studies that used RCT designs. Five of them used melatonin, and one study used an antipsychotic. In studies using melatonin, a meta-analysis revealed that there was a large effect size of using melatonin in reducing sleep latency in children and adolescents with ASD. The overall effect size was -0.87 (95\% confidence interval [CI]) -1.11, $0.63, Z=7.11 \quad(P<0.00001)$. In terms of antipsychotics, our review revealed that the antipsychotic resperidon was safe and effective in managing sleep problems in children and adolescents with ASD. Both melatonin and resperidon improved sleep latency and reduced night-time awakenings. However, mild side effects were reported in a small number of participants (figure 1).
Discussion Although the quality of the evidence in these studies was moderate according to the GRADE approach, both melatonin and resperidon seem to be safe and effective in improving sleep in children and adolescents with ASD. Some side effects have been observed in a small number of children. Observing and recording side effects is crucial to control medication doses.

\section{P024 CHARACTERISING SCHOOL-AGE CHILDREN'S SLEEP IN SHAQRA PROVINCE, SAUDI ARABIA}

${ }^{1}$ Hetaf Alammar*, ${ }^{2}$ Jane Blackwell. ${ }^{1}$ Assistant Professor in Psychology at Shaqra University, Shaqra; ${ }^{2}$ Postdoctoral Researcher, Child Oriented Mental health Intervention Centre (COMIC), Leeds and York Partnership NHS Foundation Trust and The University of York, York

\subsection{6/bmiresp-2019-bssconf.24}

Introduction As no previous studies have characterised the sleep of school-age children in Shaqra Province, Saudia Arabia, the aim of the current research was to assess the frequency of behaviours associated with common paediatric sleep difficulties in this population using the Arabic version of the Children's Sleep Habits Questionnaire.

Methods The Children's Sleep Habits Questionnaire was used to measure self-reported and parent-reported bedtime resistance, sleep onset delay, sleep duration, sleep anxiety, night wakings, parasomnias, sleep disordered breathing and daytime sleepiness. ${ }^{3}$ Families were recruited through six schools and parents were asked to complete the questionnaire on behalf of children aged 7-12 years old, whereas adolescents completed the self-reported version.

Results 150 females and 139 males aged between 7-17 years old were recruited (see table 1). 92\% of the children and adolescents had a score of 41 or above indicating that they have a clinically significant sleep problem $(89 \%$ of males and 95\% of females).

Results also indicated that there was a significant difference between males and females in secondary school in total score of CSHQ and sub score (sleep duration, sleep anxiety and sleep parasomnia). In addition, there was a significant difference between males and females in intermediate school in sub score of CSHQ in bedtime resistance, sleep anxiety and sleep disorder breathing (see table 2).

Discussion The current study highlights the high prevalence of clinically significant sleep problems in this population. The results are consistent with previous research which suggests that children from Saudi Arabia sleep less than children in other countries, ${ }^{1}$ that males have longer sleep durations compared to females and that females report more daytime sleepiness compared to males. ${ }^{2}$ The results suggest that increasing

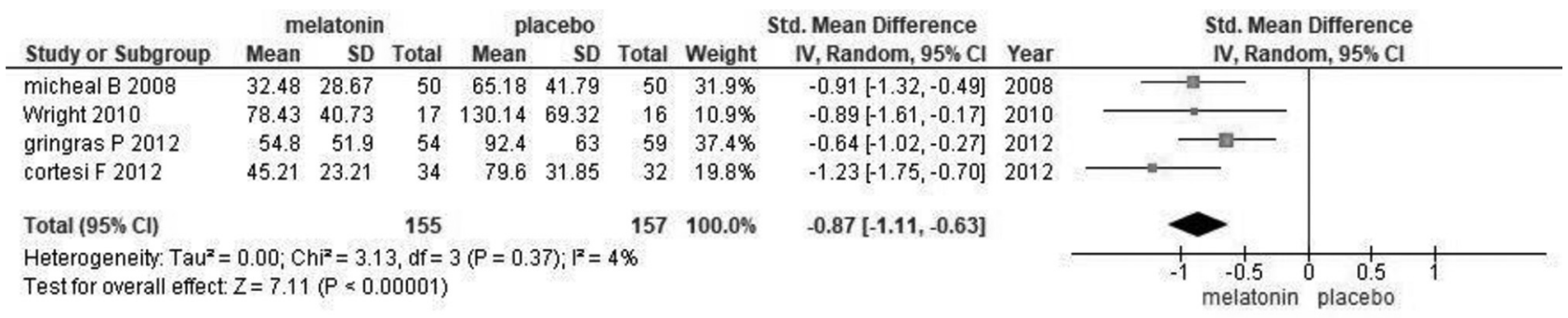

Abstract P023 Figure 1 Meta-analysis of the efficacy of using melatonin in improving sleep latency in children and adolescents with ASD 